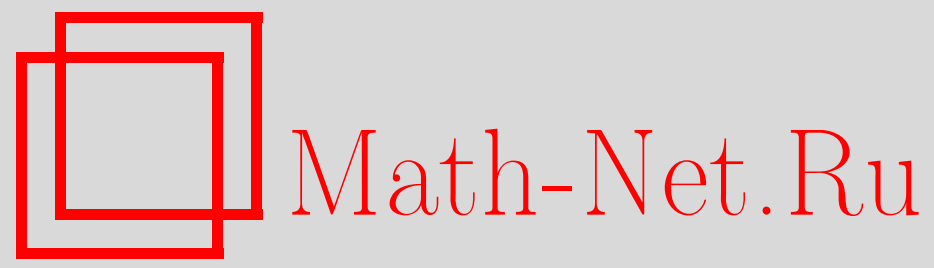

Д. М. Ермилов, О. А. Козлитин, Цикловая структура полиномиального генератора над кольцом Галуа, Матем. вопр. криптогр., 2013, том 4, выпуск 1, 27-57

DOI: https://doi.org/10.4213/mvk72

Использование Общероссийского математического портала Math-Net.Ru подразумевает, что вы прочитали и согласны с пользовательским соглашением

http://www.mathnet.ru/rus/agreement

Параметры загрузки:

IP : 54.89 .56 .158

26 апреля 2023 г., 17:43:02 


\title{
Цикловая структура полиномиального генератора над кольцом Галуа
}

\author{
Д.М.Ермилов, О.А. Козлитин \\ ООО «Центр сертификационных исследований», Москва
}

Получено 20.IV.2012

В работе исследуется цикловая структура полиномиального преобразования кольца Галуа. Полученные результаты могут быть использованы при изучении криптографических свойств выходной последовательности полиномиального генератора.

Ключевые слова: нелинейные рекуррентные последовательности, кольца Галуа, граф отображения

Cyclic structure of a polynomial generator over the Galois ring

\author{
D. M. Ermilov, O. A. Kozlitin
}

LLC "Certification Research Center", Moscow

Abstract. The cyclic structure of a polynomial generator over the Galois ring is investigated. Results may be used in the study of cryptographic properties of the output sequence of a polynomial generator.

Key words: nonlinear recurrent sequences, Galois ring, graph of the mapping

Citation: Mathematical Aspects of Cryptography, 2013, vol. 4, no. 1, pp. $27-57$ (Russian). Работа выполнена при поддержке гранта Президента РФ НШ-8.2010.10 


\section{1. Введение}

Пусть $R$ - кольцо. Один из способов выработки псевдослучайных последовательностей над $R$ состоит в использовании рекурренты с глубиной зависимости 1:

$$
\forall i \geq 0: u(i+1)=F(u(i)),
$$

где $u(0) \in R$ - начальное заполнение, а $F$ - некоторое преобразование кольца $R$. Из соображений быстродействия функция $F$ должна быть легко реализуемой. Например, можно изучать полиномиальные функции $F$ : во-первых, они строятся с помощью стандартных кольцевых операций, а во-вторых, разработан математический аппарат для их исследования. Всюду в этой работе функция $F$ полиномиальна. Там, где это не приводит к недоразумениям, будем отождествлять функцию $F$ с представляющим ее полиномом над $R$.

Первый естественный вопрос, возникающий в связи с генератором (1), - каковы дефект и период последовательности $u$. Это - частный случай вопроса о строении графа $G_{F}$ преобразования $F$. В такой формулировке (для произвольного кольца $R$ ) рассматриваемая задача далека от решения, поэтому ограничимся наиболее исследованным классом колец - конечными локальными кольцами главных идеалов. К этому классу относятся, например, все конечные поля, все примарные кольца вычетов, а также целый ряд других коммутативных и некоммутативных колец [4].

Задача о строении графа $G_{F}$ в случае, когда $R$ - примарное кольцо вычетов, рассматривалась в работах М.В. Ларина [6], В. С. Анашина [1], В.Е. Викторенкова и Д. А. Пронькина (устное сообщение) и ряда других авторов. Имеются отдельные результаты о строении графа $G_{F}$ в ситуации, когда $R$ - простейшее некоммутативное локальное кольцо главных идеалов [5]. Вместе с тем существует важный класс коммутативных колец, занимающий промежуточное положение между примарными кольцами вычетов и произвольными конечными локальными кольцами главных идеалов, - кольца Галуа. Это - минимальный класс, содержащий как все примарные кольца вычетов, так и все конечные поля [4].

Полиномиальные преобразования колец Галуа описаны в работе [7]. Задача об описании графа $G_{F}$ преобразования $F$ над произвольным кольцом Галуа $R$, насколько известно авторам, в литературе не обсуждалась. Целью настоящей работы является описание графа $G_{F}$ полиномиального преобразования $F: R \rightarrow R$ кольца Галуа $R=G R\left(q^{n}, p^{n}\right)$ мощности $q^{n}$ и характеристики $p^{n}$. 
Пусть $a \in R$. Обозначим через $t_{n}(a)$ период последовательности

$$
a, F(a), F(F(a)), \ldots,
$$

а через $t_{1}(a)-$ период последовательности

$$
\bar{a}, \bar{F}(\bar{a}), \bar{F}(\bar{F}(\bar{a})), \ldots,
$$

где $\bar{a}$ и $\bar{F}-$ результаты приведения $a$ и $F$ по модулю радикала $p R$. Перечислим полученные результаты.

1. Элемент $a \in R$ назовем цииклическим, если он лежит на некотором цикле графа $G_{F}$, и псевдоциклическим, если он сравним с циклическим элементом по модулю радикала $p R$. Среди псевдоциклических элементов $a$ выделим особые, то есть те, для которых

$$
F^{\prime}(a) \equiv 0 \quad(\bmod p R) .
$$

Показано, что для особых вершин $a$ верно равенство $t_{n}(a)=t_{1}(a)$, а для неособых возможно неравенство $t_{n}(a)>t_{1}(a)$ (теорема 1$)$.

2. В различных ситуациях найдены формулы для вычисления величины $t_{n}(a)$ (теоремы $\left.2,3,4,5,6\right)$. Показано, что имеет место достижимая оценка

$$
t_{n}(a) \leq p^{n-2}(q-1) \cdot t_{1}(a),
$$

в то время как над кольцом вычетов $\mathbb{Z}_{p^{n}}$

$$
t_{n}(a) \leq p^{n-2}(p-1) \cdot t_{1}(a)
$$

Этот результат демонстрирует новое качество, возникающее при переходе от кольца вычетов к кольцу Галуа.

3. Пусть $R_{s}=R / p^{s} R, C$ - цикл по модулю $p^{s} R$,

$$
\varphi_{S}: R_{S+1} \rightarrow R_{S}
$$

- эпиморфизм, приводящий элементы кольца $R_{s+1}$ по модулю $p^{s} R$. Теорема 7 описывает прообраз $\varphi_{s}^{-1}(C)$ цикла $C$ и позволяет вычислять цикловую структуру по модулю $p^{s+1} R$, исходя из цикловой структуры по модулю $p^{s} R$.

В следующем параграфе предложена классификация вершин графа $G_{F}$ и найдены условия принадлежности вершин различным классам.

2013. T. 4. № 1. C. $27-57$ 


\section{2. Классификация вершин}

Всюду далее $n \in \mathbb{N}, R=G R\left(q^{n}, p^{n}\right)$ - кольцо Галуа мощности $q^{n}$ и характеристики $p^{n}, I=p R-$ его радикал (см., например, [4]), $F(x) \in R[x]-$ полином, задающий преобразование кольца $R, G_{F}$ - граф этого преобразования. Если подмножество $U \subseteq R$ инвариантно относительно $F$, то $G_{F}(U)$ есть подграф графа $G$, порожденный подмножеством $U$ (см., например, [2]).

Пусть $\bar{F}(x)$ - образ полинома $F(x)$ под действием естественного эпиморфизма

$$
R \rightarrow \bar{R}=R / I .
$$

Аналогично определяются граф $G_{\bar{F}}$ и его подграф $G_{\bar{F}}(V)$, где подмножество $V \subseteq \bar{R}$ инвариантно относительно $\bar{F}$.

Вершины, лежащие на цикле, будем называть ичиклическими. Множества циклических вершин графов $G_{F}$ и $G_{\bar{F}}$ будем обозначать через $C_{F}$ и $C_{\bar{F}}$ соответственно. Обозначим через $F^{[0]}$ тождественное преобразование, а через $F^{[m]}, m \in \mathbb{N},-$ преобразование

$$
F \circ F \circ \cdots \circ F(m \text { раз }),
$$

где о - операция композищии. Имеет место

Утверждение 1. Если $a \in C_{F}$, то $\bar{a} \in C_{\bar{F}}$. Если $\bar{a} \in C_{\bar{F}}$, то существует $b \in I$ со свойством $a+b \in C_{F}$.

Доказательство. Первая часть утверждения очевидна. Докажем его вторую часть. Пусть элемент $a \in R$ таков, что $\bar{a} \in C_{\bar{F}}$. Рассмотрим компоненту связности графа $G_{F}$, содержащую вершину $a$. В этой компоненте связности есть цикл $c_{0}, c_{1}, \ldots, c_{l-1}$. Если элемент $\bar{a}$ отсутствует в ряду $\bar{c}_{0}, \bar{c}_{1}, \ldots, \bar{c}_{l-1}$, то он отсутствует и в последовательности $\bar{c}_{0}, \bar{F}\left(\bar{c}_{0}\right), \bar{F}^{[2]}\left(\bar{c}_{0}\right), \ldots$, что невозможно в силу цикличности $\bar{a}$. Следовательно, $a \equiv c_{i}(\bmod I)$ для подходящего $i \in\{0, \ldots, l-1\}$.

Семейство $C_{F}^{\#}=C_{F}+I$ назовем множеством псевдоциклических вершин. Очевидно, что всякая циклическая вершина является псевдоциклической. Расстоянием от вершины $a \in R$ до множества $C_{F}^{\#}$ назовем минимальное $m \in \mathbb{N}_{0}$ со свойством $F^{[m]}(a) \in C_{F}^{\#}$. Аналогично определяется расстояние от вершины $\bar{a} \in \bar{R}$ до множества $C_{\bar{F}}$. Для них используются обозначения $\rho\left(a, C_{F}^{\#}\right), \rho\left(\bar{a}, C_{\bar{F}}\right)$. 
Утверждение 2. Для всякого $a \in R$ справедливо равенство $\rho\left(a, C_{F}^{\#}\right)=$ $\rho\left(\bar{a}, C_{\bar{F}}\right)$.

Доказательство. Достаточно доказать, что

$$
\forall m \geq 0: \quad F^{[m]}(a) \in C_{F}^{\#} \Leftrightarrow \bar{F}^{[m]}(\bar{a}) \in C_{\bar{F}} .
$$

Импликация «слева направо» следует из первой части утверждения 1. Докажем импликацию «справа налево». Пусть элемент $b=F^{[m]}(a)$ таков, что $\bar{b} \in C_{\bar{F}}$. Это означает, что существует $l \geq 1$ со свойством $F^{[l]}(b) \equiv b(\bmod I)$. Тогда для всякого $t \geq 1$ справедливо соотношение $F^{[t \cdot l]}(b) \equiv b(\bmod I)$. Граф $G_{F}$ есть совокупность циклов с подходами. Поэтому существует $t_{0} \geq 1$ такое, что $F^{\left[t_{0} \cdot l\right]}(b) \in C_{F}$. Следовательно, $b \in C_{F}+I=C_{F}^{\#}$.

Поскольку преобразование $F$ сохраняет конгруэнции кольца $R$, множество $C_{F}^{\#}$ инвариантно относительно $F$. Поэтому можно рассматривать подграф

$$
G_{F}\left(C_{F}^{\#}\right) \leq G_{F}
$$

Утверждение 2 в определенном смысле сводит описание графа $G_{F}$ к описанию его подграфа $G_{F}\left(C_{F}^{\#}\right)$. Дальнейшие усилия направлены на описание этого подграфа.

Пусть $a \in C_{F}^{\#}$, и цикл графа $G_{\bar{F}}$, содержащий вершину $\bar{a}$, имеет длину $l$. Положим

$$
C_{F}(a)=\left\{a, F(a), \ldots, F^{[l-1]}(a)\right\} .
$$

Множество $C_{F}^{\#}(a)=C_{F}(a)+I$ назовем псевдоциклом, содержащим элемент $a$.

Утверждение 3. Если в графе $G_{\bar{F}}\left(C_{\bar{F}}\right)$ ровно $k$ цүиклов, и $\bar{a}_{1}, \bar{a}_{2}, \ldots, \bar{a}_{k}-$ их представители, то

$$
C_{F}^{\#}=\bigcup_{i=1}^{k} C_{F}^{\#}\left(a_{i}\right),
$$

причем слагаемые в разложении (2) попарно не пересекаются.

Доказательство. Ввиду утверждения 2 и инвариантности $C_{F}^{\#}$ относительно преобразования $F$ имеем

$$
\forall i \in\{1, \ldots, k\}: C_{F}\left(a_{i}\right) \subseteq C_{F}^{\#}
$$

Отсюда $C_{F}^{\#}\left(a_{i}\right) \subseteq C_{F}^{\#}$ для $i=1,2, \ldots, k$, и правая часть равенства (2) включается в его левую часть. 
Обратно, если $a \in C_{F}^{\#}$, то существует $i \in\{1, \ldots, k\}$ такое, что $\bar{a}$ и $\bar{a}_{i}$ принадлежат одному циклу графа $G_{\bar{F}}$. Если длина этого цикла равна $l$, то существует $m \in\{0, \ldots, l-1\}$ со свойством $a \equiv F^{[m]}\left(a_{i}\right)(\bmod I)$. Поэтому $a \in C_{F}\left(a_{i}\right)+I=C_{F}^{\#}\left(a_{i}\right)$. Равенство (2) доказано.

Если $b \in C_{F}^{\#}\left(a_{i}\right) \cap C_{F}^{\#}\left(a_{j}\right)$, то $\bar{b}$ лежит как на цикле, содержащем $\bar{a}_{i}$, так и на цикле, содержащем $\bar{a}_{j}$. Отсюда $i=j$.

Утверждение 3 сводит изучение графа $G_{F}\left(C_{F}^{\#}\right)$ к изучению его псевдоциклов. Выясним строение псевдоциклов. Для этого рассмотрим кольцевой аналог разложения Тейлора.

Наделим $R$ структурой $\mathbb{Z}$-бимодуля. Для многочлена $F(x) \in R[x]$ естественным образом определим его k-ю производную $F^{(k)}(x)$ (см., например, [3]).

Утверждение 4. Пусть $F(x) \in R[x] u s \in \mathbb{N}$. Тогда для любых $x, y \in R$

$$
F\left(x+p^{s} y\right)=F(x)+F^{\prime}(x) p^{s} y+\sum_{k \geq 2} H_{k}(x) p^{k s} y^{k},
$$

где для элементов $H_{k}(x) \in R$ верно равенство $F^{(k)}(x)=k ! \cdot H_{k}(x), k=$ $=2,3,4, \ldots$. получаем

Пусть $F(x)=\sum_{l \geq 0} a_{l} x^{l}$. Используя формулу бинома Ньютона,

$$
\begin{gathered}
F\left(x+p^{s} y\right)=\sum_{l \geq 0} a_{l}\left(x+p^{s} y\right)^{l}=\sum_{l \geq 0} a_{l} \sum_{k=0}^{l}\left(\begin{array}{l}
l \\
k
\end{array}\right) x^{l-k} p^{k s} y^{k}= \\
=\sum_{k \geq 0}\left[\sum_{l \geq k}\left(\begin{array}{l}
l \\
k
\end{array}\right) a_{l} x^{l-k}\right] p^{k s} y^{k} .
\end{gathered}
$$

Обозначим выражение в квадратных скобках через $H_{k}(x), k=0,1,2, \ldots$ Очевидно, что $H_{0}(x)=F(x), H_{1}(x)=F^{\prime}(x)$, и $F^{(k)}(x)=k ! \cdot H_{k}(x)$ при $k \geq 2$.

Из утверждения 4 вытекает

Следствие 1. Для любых $s \in \mathbb{N} u x, y \in R$ верно соотночение

$$
F\left(x+p^{s} y\right) \equiv F(x)+F^{\prime}(x) p^{s} y \quad\left(\bmod I^{s+1}\right) .
$$


Положим $\Gamma(R)=\left\{x \in R \mid x^{q}=x\right\}$. Согласно [4] всякий элемент $a \in R$ однозначно представляется в виде

$$
a=a_{0}+p a_{1}+p^{2} a_{2}+\cdots+p^{n-1} a_{n-1},
$$

где $a_{i} \in \Gamma(R), i=0,1, \ldots, n-1$. Такое представление называется $p$-адическим разложением элемента $a$.

Псевдоциклическую вершину $a$ графа $G_{F}$ назовем особой, если

$$
F^{\prime}(a) \equiv 0 \quad(\bmod I) .
$$

Псевдоциклические вершины, не являющиеся особыми, назовем неособыми. Псевдоцикл будем называть особым, если среди его вершин есть особые. Псевдоциклы, не являющиеся особыми, будем называть неособыми.

Псевдоциклическую вершину $b$ графа $G_{F}$ назовем регулярной, если

$$
\left|F^{-1}(b) \cap C_{F}^{\#}\right|=1 .
$$

Регулярные вершины - это псевдоциклические вершины, у которых полустепень захода в графе $G_{F}\left(C_{F}^{\#}\right)$ равна 1.

Утверждение 5. Пусть $a \in C_{F}^{\#} u b \equiv F(a)(\bmod I)$. Следующие утверждения эквивалентны:

1. а-неособая вершина.

2. $b$ - регулярная вершина.

Доказательство. Рассмотрим систему сравнений относительно $x \in R$ :

$$
\left\{\begin{array}{l}
F(x)=b \\
x \equiv a(\bmod I)
\end{array}\right.
$$

Вершина $b$ регулярна тогда и только тогда, когда система (6) имеет единственное решение. Разложение (5) элемента $x$ имеет вид

$$
x=x_{0}+p x_{1}+p^{2} x_{2}+\cdots+p^{n-1} x_{n-1},
$$

где $x_{i} \in \Gamma(R), i=0,1, \ldots, n-1$, причем в силу (6) $x_{0}=a_{0}$. Опираясь на соотношение (4), применим к многочлену $F(x)-b$ стандартный алгоритм поднятия корня по модулю радикала, позволяющий для всякого $s \in\{1, \ldots, n-$ $1\}$ восстанавливать координату $x_{s}$ по координатам $a_{0}, x_{1}, \ldots, x_{s-1}$. Это 
восстановление осуществляется однозначно тогда и только тогда, когда $F^{\prime}\left(a_{0}\right) \not \equiv 0(\bmod I)$. Поскольку $F^{\prime}(a) \equiv F^{\prime}\left(a_{0}\right)(\bmod I)$, система (6) имеет единственное решение тогда и только тогда, когда $a-$ неособая вершина. $\triangleright$

Множество всех особых псевдоциклических вершин графа $G_{F}$ обозначим через $S C_{F}^{\#}$, а множество всех неособых псевдоциклических вершин через $N S C_{F}^{\#}$. Сформулируем центральный результат этого параграфа.

Теорема 1. Справедливы следующие утверждения.

1. $N S C_{F}^{\#} \subseteq C_{F}$. B частности, компонентами связности всякого неособого псевдоцикла являются циклы без подходов.

2. Для всякой вершины $a \in S C_{F}^{\#}$ существует единственный элемент $b \in I$ со свойством $a+b \in C_{F}$. В частности, если $a \in S C_{F}^{\#}$ и $\bar{a}$ лежит на ичикле длины l, то псевдоцикл $C_{F}^{\#}(a)$ есть изикл длины l c подходами.

Доказательство. 1. Рассмотрим неособый псевдоцикл $C_{F}^{\#}(a)$. Если $x \in C_{F}^{\#}(a)$, то $\bar{x}=\bar{F}(\bar{b})$, где вершины $\bar{b}$ и $\bar{a}$ принадлежат одному циклу графа $G_{\bar{F}}$. Тогда $b \in C_{F}^{\#}(a)$ и $x \equiv F(b)(\bmod I)$. Поскольку вершина $b$ неособая, вершина $x$ регулярна (утверждение 5). Таким образом, у каждой вершины псевдоцикла $C_{F}^{\#}(a)$ полустепень захода равна 1. Следовательно, псевдоцикл $C_{F}^{\#}(a)$ целиком состоит из циклических элементов.

2. Существование элемента $b$ следует из утверждения 1 . Докажем его единственность. Рассуждаем от противного. Пусть $a \in S C_{F}^{\#}$ и существуют такие два элемента $b_{1} \neq b_{2} \in I$, что $a+b_{1} \in C_{F}$ и $a+b_{2} \in C_{F}$. Пусть $s \in \mathbb{N}-$ минимальное со свойством $b_{1} \not \equiv b_{2}\left(\bmod I^{s+1}\right)$. Ввиду (4) для некоторого $r \in R^{*}$ справедлива следующая цепочка сравнений по модулю $I^{s+1}$ :

$$
\begin{gathered}
F^{[s]}\left(a+b_{1}\right) \equiv F^{[s]}\left(a+b_{2}+p^{s} r\right) \equiv F^{[s]}\left(a+b_{2}\right)+\left(F^{[s]}\right)^{\prime}(a) p^{s} r= \\
=F^{[s]}\left(a+b_{2}\right)+F^{\prime}(a)\left(F^{[s-1]}\right)^{\prime}(F(a)) p^{s} r \equiv F^{[s]}\left(a+b_{2}\right) .
\end{gathered}
$$

Вершины $a+b_{1}$ и $a+b_{2}$ являются циклическими по модулю $I^{s+1}$. Поэтому

$$
a+b_{1} \equiv a+b_{2} \quad\left(\bmod I^{s+1}\right) .
$$

Получено противоречие.

Покажем, что в псевдоцикле $C_{F}^{\#}(a)$ имеется единственный цикл. От противного: если в этом псевдоцикле есть по крайней мере 2 цикла, то по модулю радикала $I$ они состоят из одних и тех же элементов. Тогда вершина $a$ 
достраивается до циклической по крайней мере двумя способами, то есть существуют разные $b_{1}, b_{2} \in I$ такие, что $a+b_{1} \in C_{F}$ и $a+b_{2} \in C_{F}$. Противоречие с первой частью пункта 2.

Теперь покажем, что единственный цикл, входящий в псевдоцикл $C_{F}^{\#}(a)$, имеет длину $l$. От противного: если длина этого цикла больше $l$, то на цикле графа $G_{\bar{F}}$, содержащем элемент $\bar{a}$, есть элемент $\bar{\alpha}$ такой, что вершина $\alpha \in R$ достраивается до циклической по крайней мере двумя способами. Получено противоречие. $\triangleright$

Пусть $S C_{\bar{F}}=\left\{\bar{a} \mid a \in S C_{F}^{\#}\right\}$.

Следствие 2. Во введенных обозначениях справедливо равенство

$$
\left|C_{F}\right|=\left|C_{F}^{\#}\right|-\left|S C_{\bar{F}}\right| \cdot(|I|-1) .
$$

Доказательство. Если $\bar{a} \in C_{\bar{F}} \backslash S C_{\bar{F}}$, то а достраивается до циклической вершины $|I|$ способами. Если $\bar{a} \in S C_{\bar{F}}$, то a достраивается до циклической вершины единственным способом. Поэтому

$$
\left|C_{F}\right|=\left(\left|C_{\bar{F}}\right|-\left|S C_{\bar{F}}\right|\right) \cdot|I|+\left|S C_{\bar{F}}\right|=\left|C_{\bar{F}}\right| \cdot|I|-\left|S C_{\bar{F}}\right| \cdot(|I|-1) .
$$

Осталось заметить, что

$$
\left|C_{F}^{\#}\right|=\left|C_{F}+I\right|=\left|C_{\bar{F}}\right| \cdot|I| \cdot \triangleright
$$

Формула (7) позволяет вычислять количество $\left|C_{F}\right|$ циклических точек графа $G_{F}$ при условии, что задан многочлен $F(x)$ и описано множество $C_{\bar{F}}$.

\section{3. Оценка длины неособого цикла}

Назовем цикл неособым, если все его вершины являются неособыми, и особым, если среди его вершин есть особая вершина. Согласно пункту 2 теоремы 1 длина особого цикла $C$ совпадает с длиной его образа $\bar{C}$ под действием естественного эпиморфизма $R \rightarrow \bar{R}$. Поэтому всюду далее обсуждаются неособые циклы.

Рассмотрим неособый цикл, содержащий элемент $a \in R$. Если привести этот цикл по модулю $I^{s}$, то получится цикл длины $t_{s}(a), s=1,2, \ldots, n$. Иными словами, $t_{s}(a)$ - это минимальное натуральное $t$ со свойством

$$
F^{[t]}(a) \equiv a \quad\left(\bmod I^{S}\right) .
$$

2013. T. 4. № 1. C. 27-57 
В тех случаях, когда это не приводит к недоразумениям, вместо $t_{s}(a)$ будем писать $t_{s}, s=1,2, \ldots, n$. Очевидно, что $t_{s} \mid t_{s+1}$ для всякого $s \in\{1, \ldots, n-1\}$. Цель дальнейших усилий - исследовать последовательность

$$
t_{1}, t_{2}, \ldots, t_{n}
$$

Поскольку вершина $a$ принадлежит неособому циклу, для любого $t \geq 1$ имеем

$$
\left(F^{[t]}\right)^{\prime}(a)=\prod_{i=0}^{t-1} F^{\prime}\left(F^{[i]}(a)\right) \not \equiv 0 \quad(\bmod I) .
$$

Пусть $e-$ единица кольца $R$. Если $f_{s}=F^{\left[t_{s}\right]}$, то $\alpha_{s}=f_{s}^{\prime}(a) \in R^{*}, s=$ $=1,2, \ldots, n-1$. Положим

$$
\delta_{s}= \begin{cases}p, & \text { если } \bar{\alpha}_{s}=\bar{e}, \\ \operatorname{ord} \bar{\alpha}_{s} & \text { в противном случае. }\end{cases}
$$

Утверждение 6. Для всякого $s \in\{1, \ldots, n-1\}$ верно соотношение $t_{s+1} \mid t_{s} \cdot \delta_{s}$.

Доказательство. Достаточно доказать, что

$$
f_{s}^{\left[\delta_{s}\right]}(a) \equiv a \quad\left(\bmod I^{s+1}\right) .
$$

Для некоторого $r \in R$ имеем

$$
f_{s}(a) \equiv a+p^{s} r \quad\left(\bmod I^{s+1}\right) .
$$

Применив $\left(\delta_{s}-1\right)$ раз формулу (4), получим

$$
f_{s}^{\left[\delta_{s}\right]}(a) \equiv a+p^{s} r\left(e+\alpha_{s}+\alpha_{s}^{2}+\cdots+\alpha_{s}^{\delta_{s}-1}\right) \quad\left(\bmod I^{s+1}\right) .
$$

Осталось показать, что

$$
\bar{e}+\bar{\alpha}_{s}+\bar{\alpha}_{s}^{2}+\cdots+\bar{\alpha}_{s}^{\delta_{s}-1}=\overline{0} .
$$

Если $\bar{\alpha}_{s}=\bar{e}$, то ввиду (9) $\delta_{s}=p$, и равенство (11) очевидно. Если $\bar{\alpha}_{s} \neq \bar{e}$, то сумма в левой части (11) равна

$$
\left(\bar{\alpha}_{s}^{\delta_{s}}-e\right)\left(\bar{\alpha}_{s}-e\right)^{-1}=\overline{0} .
$$

Таким образом, соотношение (10) доказано. $\triangleright$

Из утверждения 6 вытекает соотношение

$$
t_{n}(a)=t_{1}(a) \cdot \Delta(a), \quad \text { где } \quad \Delta(a) \mid \delta_{1} \delta_{2} \ldots \delta_{n-1} .
$$

Его можно рассматривать как верхнюю оценку длины неособого цикла.

Уточним оценку (12). Сначала исследуем величины $\delta_{s}, s=1,2, \ldots, n-1$. Положим $d_{s}=t_{s+1} / t_{s}$. 
Утверждение 7. Если $j \geq 1 u \delta_{j}=p$, то $\delta_{s}=p$ для всякого $s \geq j$.

Доказательство. Условия $\delta_{j}=p$ и $f_{j}^{\prime}(a) \equiv e(\bmod I)$ равносильны. Индукцией по $s \geq j$ докажем, что

$$
f_{s}^{\prime}(a) \equiv e \quad(\bmod I) .
$$

База индукции верна по условию. Осуществим индуктивный переход от $s$ к $s+1$. Пусть $s \geq j$ и $f_{s}^{\prime}(a) \equiv e(\bmod I)$. Так как

$$
f_{s}(a) \equiv a \quad\left(\bmod I^{s}\right),
$$

то $f_{s}(a) \equiv a(\bmod I)$. Отсюда и по предположению индукции имеем

$$
f_{s+1}^{\prime}(a)=\left(f_{s}^{\left[d_{s}\right]}\right)^{\prime}(a)=\prod_{i=0}^{d_{s}-1} f_{s}^{\prime}\left(f_{s}^{[i]}(a)\right) \equiv\left[f_{s}^{\prime}(a)\right]^{d_{s}} \equiv e \quad(\bmod I) . \quad \triangleright
$$

Теперь перейдем к изучению величин $d_{s}, s=1,2, \ldots, n-1$. Далее будет использоваться следующий факт, вытекающий из разложения Тейлора.

Лемма 1. Пусть $s \geq 2 u$

$$
f_{s}(a) \equiv a+p^{s} y \quad\left(\bmod I^{s+2}\right) .
$$

Тогда для всякого $j \geq 1$ верно соотношение

$$
f_{s}^{[j]}(a) \equiv a+p^{s} y \sum_{k=0}^{j-1} \alpha_{s}^{k} \quad\left(\bmod I^{s+2}\right) .
$$

Доказательство. Используется индукция по $j$. База индукции $(j=1)$ очевидна. Осуществим индуктивный переход от $j$ к $j+1$.

Поскольку $s \geq 2$, имеем $2 s \geq s+2$. В силу (3) верно соотношение

$$
f_{s}\left(a+p^{s} x\right) \equiv f_{s}(a)+f_{s}^{\prime}(a) p^{s} x \quad\left(\bmod I^{s+2}\right) .
$$

По предположению индукции

$$
f_{s}^{[j+1]}(a)=f_{s}\left(f_{s}^{[j]}(a)\right) \equiv f_{s}\left(a+p^{s} y \sum_{k=0}^{j-1} \alpha_{s}^{k}\right) \quad\left(\bmod I^{s+2}\right) .
$$

2013. T. 4. № 1. C. 27-57 
Последовательно применяя (15) и (13), получаем цепочку сравнений по модулю $I^{s+2}$ :

$$
\begin{gathered}
f_{s}\left(a+p^{s} y \sum_{k=0}^{j-1} \alpha_{s}^{k}\right) \equiv f_{s}(a)+f_{s}^{\prime}(a) p^{s} y \sum_{k=0}^{j-1} \alpha_{s}^{k} \equiv \\
\equiv a+p^{s} y+f_{s}^{\prime}(a) p^{s} y \sum_{k=0}^{j-1} \alpha_{s}^{k} \equiv a+p^{s} y+p^{s} y \sum_{k=1}^{j} \alpha_{s}^{k} \equiv a+p^{s} y \sum_{k=0}^{j} \alpha_{s}^{k} . \quad \triangleright
\end{gathered}
$$

Введем обозначение, которое будет активно применяться в дальнейшем:

$$
\mu=\left\{\begin{array}{l}
0, \text { если } d_{1}>1, \\
\max \left\{s \mid d_{1}=d_{2}=\cdots=d_{s}=1\right\} \text { в противном случае. }
\end{array}\right.
$$

Из оценки (12) и утверждения 7 следует, что поведение величин $d_{s}$ зависит от равенства $\delta_{1}=p$. В следующем параграфе рассматривается случай $\delta_{1}=p>2$.

\section{4. Длины циклов: случай $\boldsymbol{\delta}_{1}=\boldsymbol{p}>2$}

Сформулируем центральный результат параграфа.

Теорема 2. Пусть $\delta_{1}=p>2$ и величина $\mu$ определена равенством (16). Соотночение

$$
\forall s>\mu: \quad d_{s}=p
$$

выполняется всегда, за исключением случая

$$
p=3 \quad u \quad e+y f_{1}^{\prime \prime}(a) \equiv 0 \quad(\bmod I),
$$

где у-решение уравнения

$$
f_{1}(a)-a \equiv p y \quad\left(\bmod I^{2}\right)
$$

Доказательству теоремы предпошлем три леммы.

Сначала заметим, что при $p>2$ верно неравенство $2 \bar{e} \neq 0$, то есть $2 e \in R^{*}$. Поэтому корректна запись $\frac{f_{s}^{\prime \prime}(a)}{2}$, которую следует читать как $f_{s}^{\prime \prime}(a)(2 e)^{-1}$. Используя эту запись, приведем равенство (3) по модулю $I^{s+2}$. Получим следующее утверждение. 
Лемма 2. Если $p>2 u s \geq 1$, то для всякого $x \in R$ верно соотношение

$$
f_{s}\left(a+p^{s} x\right) \equiv f_{s}(a)+f_{s}^{\prime}(a) p^{s} x+\frac{f_{s}^{\prime \prime}(a)}{2} p^{2 s} x^{2} \quad\left(\bmod I^{s+2}\right) .
$$

Заметим, что лемма 2 справедлива и в случае, когда $\delta_{1} \neq p$ (но $p>2$ ).

Лемма 3. Пусть $\delta_{1}=p>2 u$

$$
f_{1}(a) \equiv a+p y \quad\left(\bmod I^{3}\right) .
$$

Тогда для всякого $j \geq 1$ верно соотночение

$$
f_{1}^{[j]}(a) \equiv a+p y \sum_{k=0}^{j-1} \alpha_{1}^{k}+p^{2} y^{2} \frac{f_{1}^{\prime \prime}(a)}{2} \sum_{k=0}^{j-1} k^{2} \quad\left(\bmod I^{3}\right) .
$$

Доказательство. Используется индукция по $j$. База индукции $(j=1)$ очевидна. Осуществим индуктивный переход от $j$ к $j+1$.

С учетом (20) формула (19) для $s=1$ принимает вид

$$
f_{1}(a+p x) \equiv a+\left(\alpha_{1} x+y\right) p+\frac{f_{1}^{\prime \prime}(a)}{2} p^{2} x^{2} \quad\left(\bmod I^{3}\right) .
$$

По предположению индукции

$$
f_{1}^{[j+1]}(a)=f_{1}\left(f_{1}^{[j]}(a)\right) \equiv f_{1}\left(a+p y \sum_{k=0}^{j-1} \alpha_{s}^{k}+p^{2} y^{2} \frac{f_{1}^{\prime \prime}(a)}{2} \sum_{k=0}^{j-1} k^{2}\right) \quad\left(\bmod I^{3}\right)
$$

Положим

$$
x=y \sum_{k=0}^{j-1} \alpha_{1}^{k}+p y^{2} \frac{f_{1}^{\prime \prime}(a)}{2} \sum_{k=0}^{j-1} k^{2}
$$

и применим формулу (22). Получим:

$$
\begin{aligned}
f_{1}(a+p x) & \equiv a+p y+p y \sum_{k=1}^{j} \alpha_{1}^{k}+p^{2} y^{2} \alpha_{1} \frac{f_{1}^{\prime \prime}(a)}{2} \sum_{k=0}^{j-1} k^{2}+ \\
& +p^{2} y^{2} \frac{f_{1}^{\prime \prime}(a)}{2}\left(\sum_{k=0}^{j-1} \alpha_{1}^{k}\right)^{2} \quad\left(\bmod I^{3}\right) .
\end{aligned}
$$

2013. T. 4. № 1. C. 27-57 
Поскольку $\delta_{1}=p$, имеем $\alpha_{1} \equiv e(\bmod I)$. Отсюда

$$
\begin{gathered}
f_{1}^{[j+1]}(a) \equiv a+p y \sum_{k=0}^{j} \alpha_{1}^{k}+p^{2} y^{2} \frac{f_{1}^{\prime \prime}(a)}{2} \sum_{k=0}^{j-1} k^{2}+p^{2} y^{2} \frac{f_{1}^{\prime \prime}(a)}{2} j^{2} \equiv \\
\equiv a+p y \sum_{k=0}^{j} \alpha_{1}^{k}+p^{2} y^{2} \frac{f_{1}^{\prime \prime}(a)}{2} \sum_{k=0}^{j} k^{2}\left(\bmod I^{3}\right) . \quad \triangleright
\end{gathered}
$$

Лемма 4. Если $\delta_{s}=p>2$, то

$$
\sum_{k=0}^{p-1} \alpha_{s}^{k} \equiv p e \quad\left(\bmod I^{2}\right)
$$

Если известно, что $\delta_{1}=p>2$, то (23) справедливо для всякого $s \in$ $\in\{1, \ldots, n-1\}$.

Доказательство. По условию $\bar{\alpha}_{s}=\bar{e}$, то есть $\alpha_{s}=e+p h\left(\bmod I^{2}\right)$ для подходящего $h \in R$. Отсюда получаем следующую цепочку сравнений по модулю $I^{2}$ :

$$
\sum_{k=0}^{p-1} \alpha_{s}^{k} \equiv \sum_{k=0}^{p-1}(e+k p h) \equiv p e+p \cdot\left(\begin{array}{l}
p \\
2
\end{array}\right) \cdot h \equiv p e .
$$

Последнее сравнение справедливо, поскольку $p>2$. Вторая часть леммы получается, если применить утверждение 7 при $j=1$.

Доказательство теоремы 2. 1. Достаточность. Индукцией по $s \geq 1$ докажем, что если условие (18) не выполняется и $\mu<s$, то $d_{s}=p$.

Если $s=1$ и $\mu=0$, то $d_{1}=p$. Если $s=2$ и $\mu=1$, то $d_{2}=p$. Пусть $s=2$ и $\mu=0$. Тогда $d_{1}=p$, и соотношение (20) выполняется для некоторого $y \in R^{*}$. Из (21) и (23) следует, что

$$
f_{2}(a)=f_{1}^{[p]}(a) \equiv a+p^{2} y\left(e+y \frac{f_{1}^{\prime \prime}(a)}{2} \sum_{k=0}^{p-1} k^{2}\right) \quad\left(\bmod I^{3}\right) .
$$

Поскольку

$$
\sum_{k=0}^{p-1} k^{2}=\frac{(p-1) p(2 p-1)}{6}
$$


получаем, что при $p>3$

$$
f_{2}(a) \equiv a+p^{2} y \quad\left(\bmod I^{3}\right)
$$

и $d_{2}=p$. Если же $p=3$, то

$$
\sum_{k=0}^{p-1} k^{2} \equiv 2 e \quad(\bmod I)
$$

откуда с учетом (24) получаем

$$
f_{2}(a) \equiv a+p^{2} y\left(e+y f_{1}^{\prime \prime}(a)\right)\left(\bmod I^{3}\right) .
$$

Поскольку условие (18) не выполняется, $d_{2}=p$. База индукции доказана.

Осуществим индуктивный переход от $s \geq 2$ к $s+1$. Если $\mu=s$, то $d_{s+1}=p$. Если $\mu<s$, то по предположению индукции $d_{s}=p$, и соотношение (13) выполняется для некоторого $y \in R^{*}$. Из (14) и (23) следует цепочка соотношений

$$
f_{s+1}(a)=f_{s}^{[p]}(a) \equiv a+p^{s+1} y \quad\left(\bmod I^{s+2}\right) .
$$

Отсюда $d_{s+1}=p$. Индуктивный переход завершен.

2. Необходимость. Если условие (18) выполняется, то $\mu=0$, и, согласно (25), $d_{2}=1$. Получено противоречие с (17). $\triangleright$

При условии (17) величина $\Delta(a)$ из оценки (12) принимает вид

$$
\Delta(a)=p^{n-\mu-1} .
$$

Следствие 3. Пусть $\delta_{1}=p>2$. Соотномение

$$
t_{n}(a)=t_{1}(a) \cdot p^{n-\mu-1}
$$

выполняется всегда, за исключением случая (18).

В этом параграфе оценка (12) была уточнена в случае $\delta_{1}=p>2$. В следующем параграфе будет рассматриваться ситуация $\delta_{1} \neq p, p>2$. 


\section{5. Длины циклов: случай $\delta_{1} \neq p, p>2$}

Здесь, как и в предыдущем параграфе, $p>2$, однако $\delta_{1} \neq p$. Цикл, которому принадлежит вершина $a \in R$, по-прежнему является неособым.

Утверждение 8. Если $p>2, j \geq 2, d_{j}=p$, то $d_{s}=p$ для всякого $s \geq j$.

Доказательство. Используется индукция по $s \geq j$.

База индукции очевидна.

Осуществим индуктивный переход от $s \geq j$ к $s+1$. По предположению индукции $d_{s}=p$. Тогда $\delta_{s}=p$ и верно соотношение

$$
f_{s}(a) \equiv a+p^{s} y \quad\left(\bmod I^{s+2}\right),
$$

где $y \in R^{*}$. Ввиду (14) и (23) получаем

$$
f_{s+1}(a)=f_{s}^{[p]}(a) \equiv a+p^{s} y \sum_{k=0}^{p-1} \alpha_{s}^{k} \equiv a+p^{s+1} y \quad\left(\bmod I^{s+2}\right) .
$$

Отсюда $d_{s+1}>1$. Согласно утверждению 7 и поскольку $d_{s+1} \mid \delta_{s+1}$, верна цепочка импликаций

$$
\left(\delta_{s}=p\right) \Rightarrow\left(\delta_{s+1}=p\right) \Rightarrow\left(d_{s+1}=p\right) . \quad \triangleright
$$

Утверждение 9. Если $p>2, \delta_{1} \neq p u$

$$
t_{1}=\cdots=t_{i}<t_{i+1}=\cdots=t_{j}<t_{j+1},
$$

$\operatorname{mo~}_{i}=\delta_{1} u d_{s}=p$ для всякого $s \geq j$.

Доказательство. Покажем, что $d_{j}=p$. Это равенство эквивалентно системе

$$
\begin{cases}f_{j}^{\prime}(a) \equiv e & (\bmod I) \\ f_{j}(a) \neq \equiv a & \left(\bmod I^{j+1}\right) .\end{cases}
$$

Второе ограничение системы (26) следует из неравенства $t_{j}<t_{j+1}$. Докажем ее первое ограничение.

Аналогично лемме 1 можно показать, что если для некоторого $y \in R^{*}$ верно соотношение

$$
f_{1}(a) \equiv a+p^{i} y \quad\left(\bmod I^{i+1}\right),
$$

то для всякого $d \geq 1$

$$
f_{1}^{[d]}(a) \equiv a+p^{i} y \sum_{k=0}^{d-1} \alpha_{1}^{k} \quad\left(\bmod I^{i+1}\right) .
$$


Учитывая, что $\bar{\alpha}_{1} \neq \bar{e}$, получаем

$$
\forall d \geq 1: f_{1}^{[d]}(a) \equiv a+\frac{\alpha_{1}^{d}-e}{\alpha_{1}-e} \cdot p^{i} y \quad\left(\bmod I^{i+1}\right) .
$$

Ввиду того, что $f_{1}=f_{2}=\cdots=f_{i}$, имеем

$$
\forall d \geq 1: f_{i}^{[d]}(a) \equiv a+\frac{\alpha_{1}^{d}-e}{\alpha_{1}-e} \cdot p^{i} y \quad\left(\bmod I^{i+1}\right) .
$$

Отсюда $d_{i}=\delta_{1}$.

Поскольку $t_{j}=t_{i+1}$, имеем цепочку равенств и сравнений по модулю $I$

$$
f_{j}^{\prime}(a)=\left(f_{1}^{\left[\delta_{1}\right]}\right)^{\prime}(a)=\prod_{k=0}^{\delta_{1}-1} f_{1}^{\prime}\left(f_{1}^{[k]}(a)\right) \equiv \alpha_{1}^{\delta_{1}} \equiv e .
$$

Таким образом, $d_{j}=p$, и по утверждению 8 имеем

$$
\forall s \geq j: d_{s}=p . \triangleright
$$

Из утверждения 9 вытекает следующее утверждение.

Следствие 4. Если $p>2$ и $\delta_{1} \neq p$, то последовательность

$$
d_{1}, d_{2}, \ldots, d_{n-1}
$$

имеет один из видов:

a) $1,1, \ldots, 1$;

b) $1,1, \ldots, 1, \delta_{1}$;

c) $1,1, \ldots, 1, \delta_{1}, 1,1, \ldots, 1$;

d) $1,1, \ldots, 1, \delta_{1}, 1,1, \ldots, 1, p, p, \ldots, p$.

В каждом случае первая серия единии может отсутствовать.

Исследуем условия, при которых имеют место ситуации а), b), с) и d). Наиболее простой с этой точки зрения является ситуация а).

Утверждение 10. Пусть неособый элемент а таков, что $\delta_{1} \neq p$. Тогда существует единственный элемент $a^{*} \in R$ такой, что $\bar{a}=\bar{a}^{*} u t_{n}\left(a^{*}\right)=$ $=t_{1}\left(a^{*}\right)$. Ситуачия а) имеет место тогда и только тогда, когда $a=a^{*}$.

Доказательство. Достаточно доказать существование и единственность элемента $a^{*}$, обладающего указанными свойствами. 
Докажем, что для всякого $s \in\{1, \ldots, n\}$ существует $a_{s} \in R$ со свойствами $\bar{a}_{s}=\bar{a}$ и $t_{s}\left(a_{s}\right)=t_{1}\left(a_{s}\right)$.

Для этого используется индукция по $s$. База индукции: положим $a_{1}=a$. Осуществим индуктивный переход от $s \geq 1$ к $s+1$. Для всякого $x \in R$ положим $a_{s}(x)=a_{s}+p^{s} x$. Рассмотрим уравнение

$$
t_{s+1}\left(a_{s}(x)\right)=t_{1}\left(a_{s}(x)\right) \text {. }
$$

Оно равносильно уравнению

$$
f_{1}\left(a_{s}(x)\right) \equiv a_{s}(x)\left(\bmod I^{s+1}\right) .
$$

Согласно (4) последнее уравнение переписывается в виде

$$
f_{1}\left(a_{s}\right)+p^{s} \alpha_{1} x \equiv a_{s}+p^{s} x \quad\left(\bmod I^{s+1}\right),
$$

то есть

$$
f_{1}\left(a_{s}\right)-a_{s} \equiv p^{s}\left(e-\alpha_{1}\right) x \quad\left(\bmod I^{s+1}\right) .
$$

По предположению индукции $f_{1}\left(a_{s}\right) \equiv a_{s}\left(\bmod I^{s}\right)$. Поскольку $\bar{\alpha}_{1} \neq \bar{e}$, уравнение (28) имеет во множестве $\Gamma(R)$ единственное решение $x_{s}$. Индуктивный переход осуществлен.

Существование элемента $a^{*}=a_{n}$ доказано. В ходе описанного выше построения было показано, что для всякого элемента $a \in R$ существует единственный элемент

$$
b=p x_{1}+p^{2} x_{2}+\cdots+p^{n-1} x_{n-1} \in I
$$

такой, что

$$
t_{n}(a+b)=t_{1}(a+b)
$$

Поэтому элемент $a^{*}$ с указанными в условии свойствами единствен. $\triangleright$

Заметим, что при доказательстве утверждения 10 условие $p>2$ не использовалось. Следовательно, это утверждение справедливо для любого простого $p$.

Для краткости всюду далее в этом параграфе $f=f_{1}, \alpha=\alpha_{1}, \beta=$ $=f^{\prime \prime}(a), \delta=\delta_{1}$. Напомним (см., например, [7]), что нормой $\|x\|$ элемента $x \in R$ называется наибольшее $k \in\{0, \ldots, n\}$ со свойством $x \in I^{k}$. 
Теорема 3. Пусть $p>2, \delta \neq p$ и выполняются следующие условия:

1) $a \neq a^{*}$ us $=\|f(a)-a\|$;

2) $\left\|\alpha^{\delta}-e\right\| \neq s+\|\beta\|$;

3) $\nu=\min \left\{\left\|\alpha^{\delta}-e\right\|, s+\|\beta\|\right\}<2 s$.

Тогда:

1) если $s+\nu \geq n$, то имеет место одна из ситуаций b) или с) следствия 4. $\Pi$ пи этом $t_{n}=t_{1} \cdot \delta$;

2) если $s+\nu<n$, то имеет место ситуащия d) $u t_{n}=t_{1} \cdot \delta \cdot p^{n-s-\nu}$.

Доказательство. Приведем равенство (3) по модулю $I^{3 s}$ и заменим $H_{2}(a)$ на $\frac{\beta}{2}$. Получим:

$$
\forall x \in R: \quad f\left(a+p^{s} x\right) \equiv f(a)+\alpha p^{s} x+\frac{\beta}{2} p^{2 s} x^{2} \quad\left(\bmod I^{3 s}\right) .
$$

По условию $f(a)=a+p^{s} y$ для некоторого $y \in R^{*}$. Отсюда при $A_{1}=e$ и $B_{1}=0$ имеем

$$
f(a) \equiv a+p^{s} y A_{1}+p^{2 s} y^{2} \frac{\beta}{2} B_{1} \quad\left(\bmod I^{3 s}\right) .
$$

Пусть теперь при некоторых $j \geq 1$ и $A_{j}, B_{j} \in R$ верно соотношение

$$
f^{[j]}(a) \equiv a+p^{s} y A_{j}+p^{2 s} y^{2} \frac{\beta}{2} B_{j} \quad\left(\bmod I^{3 s}\right) .
$$

Используя формулу (29), получаем цепочку сравнений по модулю $I^{3 s}$

$$
\begin{gathered}
f^{[j+1]}(a)=f\left(f^{[j]}(a)\right) \equiv f\left(a+p^{s} y\left(A_{j}+p^{s} y \frac{\beta}{2} B_{j}\right)\right) \equiv \\
\equiv a+p^{s} y\left(e+\alpha A_{j}\right)+p^{2 s} y^{2} \frac{\beta}{2}\left(\alpha B_{j}+A_{j}^{2}\right) .
\end{gathered}
$$

Если положить

$$
\left\{\begin{array}{l}
A_{1}=e, B_{1}=0, \\
A_{j+1}=e+\alpha A_{j}, \\
B_{j+1}=\alpha B_{j}+A_{j}^{2},
\end{array}\right.
$$

то сравнение (30) будет выполняться для всякого $j \geq 1$. 
Непосредственно проверяется, что последовательности

$$
A_{j}=\frac{\alpha^{j}-e}{\alpha-e}, \quad j=1,2, \ldots,
$$

и

$$
B_{j}=\sum_{i=0}^{j-2}\left(\frac{\alpha^{i+1}-e}{\alpha-e}\right)^{2} \cdot \alpha^{j-2-i}, \quad j=2,3, \ldots,
$$

являются решениями рекуррентных соотношений (31). Вычислим $\left\|A_{\delta}\right\|$ и $\left\|B_{\delta}\right\|$. Очевидно,

$$
\left\|A_{\delta}\right\|=\left\|\frac{\alpha^{\delta}-e}{\alpha-e}\right\|=\left\|\alpha^{\delta}-e\right\| .
$$

Чтобы вычислить $\left\|B_{\delta}\right\|$, преобразуем выражение для $B_{j}$ :

$$
B_{j}=(\alpha-e)^{-2} \sum_{i=0}^{j-2}\left(\alpha^{j+i}-2 \alpha^{j-1}+\alpha^{j-2-i}\right) .
$$

Суммируя геометрические прогрессии, получаем

$$
B_{j}=\alpha^{-1}(\alpha-e)^{-2}\left[\left(\alpha^{j}+e\right) \frac{\alpha^{j}-\alpha}{\alpha-e}-2(j-1) \alpha^{j}\right] .
$$

Отсюда вытекает следующая цепочка равенств и сравнений по модулю $I$ :

$$
\alpha(\alpha-e)^{2} B_{\delta} \equiv-2 e+2 e-2 \delta e=-2 \delta e .
$$

Поскольку $\|2 \delta e\|=0$, имеем $\left\|B_{\delta}\right\|=0$.

Подставив в (30) $j=\delta$, получим

$$
f^{[\delta]}(a) \equiv a+p^{s} y\left(A_{\delta}+p^{s} y \frac{\beta}{2} B_{\delta}\right) \quad\left(\bmod I^{3 s}\right) .
$$

Ввиду (32) и условий 2), 3) получаем, что

$$
\left\|f^{[\delta]}(a)-a\right\|=s+\nu .
$$

Поэтому в силу утверждения 9

$$
\begin{gathered}
d_{1}=\cdots=d_{s-1}=1, d_{s}=\delta, d_{s+1}=\cdots=d_{s+\nu-1}=1, \\
d_{s+\nu}=d_{s+\nu+1}=\cdots=d_{n-1}=p,
\end{gathered}
$$

откуда вытекают требуемые равенства. $\triangleright$

В этом и предыдущем параграфах рассматривался случай $p>2$. Теперь перейдем к случаю $p=2$. 


\section{6. Длины циклов: случай $\boldsymbol{\delta}_{1}=\boldsymbol{p}=2$}

В этом и следующем параграфах $p=2$. В этом параграфе $\delta_{1}=2$, то есть $\bar{\alpha}_{1}=\bar{e}$. Как и раньше, величина $\mu$ определена равенством (16), а цикл, которому принадлежит вершина $a \in R$, является неособым. Сначала рассмотрим случай $\mu>0$.

Теорема 4. Если $\delta_{1}=p=2 u \mu>0$, то последовательность (27) имеет один из видов:

a) $1,1, \ldots, 1$;

b) $1,1, \ldots, 1,2,2, \ldots, 2$;

c) $1,1, \ldots, 1,2,1,1, \ldots, 1$;

d) $1,1, \ldots, 1,2,1,1, \ldots, 1,2,2, \ldots, 2$.

Случаи с) $и$ d) возможны лишь при условии $\alpha_{1} \equiv 3 e\left(\bmod I^{2}\right)$.

Доказательству теоремы предпошлем две леммы.

Лемма 5. Пусть $s \geq 1 u \delta_{s}=p=2$. Для всякого $k \geq 0$ верно соотношение

$$
\left(f_{s}^{\left[2^{k}\right]}\right)^{\prime}(a) \equiv \alpha_{s}^{2^{k}} \quad\left(\bmod I^{s+1}\right) .
$$

Доказательство. Для краткости положим $G_{k, s}(x)=\left(f_{s}^{\left[2^{k}\right]}\right)^{\prime}(x), k=$ $=0,1,2, \ldots$ Соотношение (33) докажем индукцией по $k \geq 0$. База индукции $(k=0)$ очевидна. Осуществим индуктивный переход от $k$ к $k+1$.

Пусть рекуррента $u \in R^{\infty}$ задана законом рекурсии (1) и начальным значением $u(0)=a$. Многократно применяя формулу производной сложной функции и используя соотношение $u(i)=F^{[i]}(u(0)), i=0,1,2, \ldots$, получаем

$$
\begin{gathered}
G_{k+1, s}^{\prime}(a)=\prod_{i=0}^{2^{k+1} t_{s}-1} F^{\prime}(u(i))=\prod_{i=0}^{2^{k} t_{s}-1} F^{\prime}(u(i)) \times \prod_{j=2^{k} t_{s}}^{2^{k+1} t_{s}-1} F^{\prime}(u(j))= \\
=G_{k, s}^{\prime}(u(0)) \cdot G_{k, s}^{\prime}\left(u\left(2^{k} t_{s}\right)\right) .
\end{gathered}
$$

Так как $\delta_{s}=p=2$, то по утверждению 7 имеем $\delta_{j}=2$ для всякого $j \geq s$. Отсюда $t_{s+k} \mid t_{s} \cdot 2^{k}$, и существует элемент $y \in R$ со свойством

$$
u\left(2^{k} t_{s}\right)=u(0)+2^{s+k} y
$$

Выпишем формулу (4) для многочлена $G_{k, s}^{\prime}(x)$ и элемента $u\left(2^{k} t_{s}\right)$ :

$$
\begin{gathered}
G_{k, s}^{\prime}\left(u\left(2^{k} t_{s}\right)\right)=G_{k, s}^{\prime}\left(u(0)+2^{k+s} y\right) \equiv \\
\equiv G_{k, s}^{\prime}(u(0))+G_{k, s}^{\prime \prime}(u(0)) \cdot 2^{k+s} y \quad\left(\bmod I^{k+s+1}\right) .
\end{gathered}
$$


Применим утверждение 4 к многочлену $G_{k, s}(x)$. Существует такой многочлен $H_{2}(x) \in R[x]$, что $G_{k, s}^{\prime \prime}(x)=2 H_{2}(x)$. Отсюда $G_{k, s}^{\prime \prime}(u(0)) \equiv 0(\bmod I)$, и ввиду (35) имеем

$$
G_{k, s}^{\prime}\left(u\left(2^{k} t_{s}\right)\right) \equiv G_{k, S}^{\prime}(u(0)) \quad\left(\bmod I^{k+s+1}\right) .
$$

В частности,

$$
G_{k, s}^{\prime}\left(u\left(2^{k} t_{s}\right)\right) \equiv G_{k, s}^{\prime}(u(0))\left(\bmod I^{s+1}\right) .
$$

Используя (34), (36) и предположение индукции, получаем цепочку равенств и сравнений по модулю $I^{s+1}$ :

$$
G_{k+1, s}^{\prime}(a)=G_{k, s}^{\prime}(u(0)) \cdot G_{k, s}^{\prime}\left(u\left(2^{k} t_{s}\right)\right) \equiv\left[G_{k, s}^{\prime}(a)\right]^{2} \equiv \alpha_{s}^{2^{k+1}} \cdot \triangleright
$$

Лемма 6. Если $\delta_{1}=p=2 u \mu>0$, то импликация

$$
\left(d_{j}=2\right) \Rightarrow\left(d_{j}=d_{j+1}=\cdots=d_{n-1}=2\right)
$$

верна для всякого $j \geq \mu+2$. Для $j=\mu+1$ импликация (37) верна тогда и только тогда, когда $\alpha_{1} \not \equiv 3 e\left(\bmod I^{2}\right)$.

Доказательство. Пусть $d_{j}=2$ и $s \geq j \geq \mu+1$. Так как $\delta_{1}=p=2$, то $\delta_{i}=2$ для всякого $i \geq 1$ (утверждение 7). Тогда $t_{s+1} \in\left\{t_{s}, 2 t_{s}\right\}$.

Если $d_{s}=2$, то $t_{s+1}=2 t_{s}$ и существует $y \in R^{*}$ со свойством

$$
f_{s}(a) \equiv a+2^{s} y \quad\left(\bmod I^{s+2}\right) .
$$

Согласно (14)

$$
f_{s+1}(a)=f_{s}^{[2]}(a) \equiv a+2^{s} y\left(e+\alpha_{s}\right) \quad\left(\bmod I^{s+2}\right) .
$$

Если $j \geq \mu+2$, то $s \geq \mu+2$ и $t_{s}>t_{1}$. Тогда $t_{s}=2^{k} t_{1}$ для некоторого $k \geq 1$. Поскольку $\delta_{1}=p=2$, имеем $\alpha_{1}=e+2 r$ для некоторого $r \in R$. По лемме 5 верна цепочка равенств и сранений по модулю $I^{2}$ :

$$
\alpha_{s}=f_{s}^{\prime}(a)=\left(f_{1}^{\left[2^{k}\right]}\right)^{\prime}(a) \equiv \alpha_{1}^{2^{k}}=(e+2 r)^{2^{k}}=\left(e+4 r+4 r^{2}\right)^{2^{k-1}} \equiv e .
$$

Отсюда и из (39) получаем: $f_{s+1}(a) \equiv a+2^{s+1} y\left(\bmod I^{s+2}\right)$. Поэтому $t_{s+2}>$ $>t_{s+1}$ и $d_{s+1}=2$. Итак, для $j \geq \mu+2$ импликация (37) доказана.

Теперь рассмотрим случай $j=\mu+1$. Пусть $s \geq j$. Если $s \geq \mu+2$, то, как показано выше, из равенства $d_{s}=2$ следует равенство $d_{s+1}=2$. Пусть 
$s=\mu+1$. По определению величины $\mu$ верно равенство $d_{s}=2$. Исследуем значение $d_{s+1}$.

Подставив $\alpha_{1}=e+2 r$ в (39), получим

$$
f_{s+1}(a) \equiv a+2^{s+1} y(e+r) \quad\left(\bmod I^{s+2}\right) .
$$

Отсюда

$$
d_{s+1}=2 \Leftrightarrow e+r \not \equiv 0 \quad(\bmod I) \Leftrightarrow \alpha_{1} \not \equiv 3 e\left(\bmod I^{2}\right) . \quad \triangleright
$$

Доказательство теоремы 4. По условию $\mu>0$. Случай а) имеет место тогда и только тогда, когда $\mu=n-1$.

Пусть $0<\mu<n-1$. Тогда $d_{\mu+1}=2$. Если $\alpha_{1} \not \equiv 3 e\left(\bmod I^{2}\right)$, то по лемме 6 равенство $d_{s}=2$ справедливо для всякого $s \geq \mu+1$. Следовательно, имеет место случай $b$ ).

Если $\alpha_{1} \equiv 3 e\left(\bmod I^{2}\right)$, то, как показано при обосновании леммы 6 , для всякого $s \geq \mu+2$ верна импликация

$$
d_{s}=2 \Rightarrow d_{s+1}=2
$$

однако $d_{\mu+2}=1$. Если $\mu=n-2$, то имеет место ситуация b). Если $\mu<n-2$, то имеем случай с) или случай d). $\triangleright$

Далее будет рассматриваться случай $\mu=0$. Сначала докажем один вспомогательный результат. $s \geq 1$.

Лемма 7. Пусть $d_{1}=\delta_{1}=p=2 u d_{2}=2$. Тогда $d_{s}=2$ для всякого

Доказательство. Используется индукция по $s \geq 2$. База индукции $(s=2)$ очевидна. Осуществим индуктивный переход от $s \geq 2$ к $s+1$.

По предположению индукции $d_{1}=d_{2}=\cdots=d_{s}=2$, то есть $t_{s+1}=2 t_{s}$ и $t_{s}=2^{s-1} t_{1}$. По лемме 5

$$
\alpha_{s}=\left(f_{1}^{\left[2^{s-1}\right]}\right)^{\prime}(a) \equiv \alpha_{1}^{2^{s-1}}\left(\bmod I^{2}\right)
$$

и, поскольку $\alpha_{1}=e+2 r$ для некоторого $r \in R$, имеем

$$
\alpha_{s} \equiv e\left(\bmod I^{2}\right)
$$

Так как $t_{s+1}=2 t_{s}$, то существует $y \in R^{*}$, для которого выполняется (38). Но тогда ввиду (39) и (40) $t_{s+2}=2 t_{s+1}$, то есть $d_{s+1}=2$.

Справедлив следующий критерий. 
Теорема 5. Пусть $\delta_{1}=p=2, \mu=0$, значения $c \in R^{*} u r, h \in R$ удовлетворяют соотношениям

$$
f_{1}(a)=a+2 c, \quad f_{1}^{\prime}(a)=e+2 r, \quad f_{1}^{\prime \prime}(a)=2 h .
$$

Соотношение $d_{1}=d_{2}=\cdots=d_{n-1}=2$ выполняется тогда и только тогда, когда

$$
r+c h \not \equiv e \quad(\bmod I)
$$

В случае, когда $n \geq 3$ и (41) не выполняется, последовательность (27) имеет вид

$$
2,1,1, \ldots, 1 \text { или } 2,1,1, \ldots, 1,2,2, \ldots, 2 .
$$

Доказательство. Поскольку $\mu=0$, верно равенство $d_{1}=2$. Тогда $t_{2}=2 t_{1}$, и ввиду разложения (3) имеем цепочку равенств и сравнений по модулю $I^{3}$ :

$$
\begin{aligned}
f_{2}(a) & =f_{1}\left(f_{1}(a)\right)=f_{1}(a+2 c) \equiv f_{1}(a)+f_{1}^{\prime}(a) 2 c+f_{1}^{\prime \prime}(a) 2 c^{2}= \\
& =a+2 c+(e+2 r) 2 c+4 h c^{2}=a+4 c(e+r+c h) .
\end{aligned}
$$

Учитывая, что $c \in R^{*}$, получаем

$$
d_{2}=2 \Leftrightarrow e+r+c h \not \equiv 0 \quad(\bmod I),
$$

то есть равенство $d_{2}=2$ эквивалентно условию (41). С другой стороны, по лемме 7 равенство $d_{2}=2$ эквивалентно цепочке равенств

$$
d_{1}=d_{2}=\cdots=d_{n-1}=2 \text {. }
$$

Первое утверждение теоремы доказано.

Докажем второе утверждение. Пусть условие (41) не выполняется. Тогда $d_{1}=2$ и $d_{2}=1$. Пусть $s \geq 3$ и $d_{s}=2$. Для завершения доказательства теоремы осталось показать, что $d_{s+1}=2$. Очевидно, $t_{s+1}=2 t_{s}$ и $t_{s}=2^{k} t_{1}$ для некоторого $k \geq 1$. Используя лемму 5 , получаем, что $\alpha_{s} \equiv e\left(\bmod I^{2}\right)$. С другой стороны, для некоторого $y \in R^{*}$ выполняется (38), а значит, и (39). Из (39) и соотношения $\alpha_{s} \equiv e\left(\bmod I^{2}\right)$ следует, что $t_{s+2}>t_{s+1}$, то есть $d_{s+1}=2$. $\triangleright$

Теоремы 4 и 5 являются «двоичными» аналогами теоремы 2, а условие (41) - аналогом условия (18).

Осталось рассмотреть последний случай: $\delta_{1} \neq p, p=2$. 


\section{7. Длины циклов: случай $\delta_{1} \neq p, p=2$}

Пусть $\delta_{1} \neq p, p=2$, вершина $a \in R$ принадлежит неособому циклу полиномиального преобразования $F$.

Лемма 8. Если $\delta_{1} \neq p, p=2 u$

$$
t_{1}=\cdots=t_{i}<t_{i+1}=\cdots=t_{j}<t_{j+1},
$$

$\bmod _{i}=\delta_{1} u d_{j}=\delta_{j}=2$.

Доказательство. Поскольку $t_{i+1}>t_{1}$, существует такой $y \in R^{*}$, что

$$
f_{1}(a) \equiv a+2^{i} y \quad\left(\bmod I^{i+1}\right) .
$$

Аналогично лемме 1 можно показать, что для всякого $d \geq 1$

$$
f_{1}^{[d]}(a) \equiv a+2^{i} y \sum_{k=0}^{d-1} \alpha_{1}^{k} \quad\left(\bmod I^{i+1}\right) .
$$

Учитывая, что $\bar{\alpha}_{1} \neq \bar{e}$, получаем

$$
\forall d \geq 1: f_{1}^{[d]}(a) \equiv a+\frac{\alpha_{1}^{d}-e}{\alpha_{1}-e} \cdot 2^{i} y \quad\left(\bmod I^{i+1}\right) .
$$

Ввиду того, что $f_{1}=f_{2}=\cdots=f_{i}$, имеем

$$
\forall d \geq 1: f_{i}^{[d]}(a) \equiv a+\frac{\alpha_{1}^{d}-e}{\alpha_{1}-e} \cdot 2^{i} y \quad\left(\bmod I^{i+1}\right) .
$$

Отсюда $d_{i}=\delta_{1}$.

Поскольку $t_{j}=t_{i+1}$, имеем цепочку равенств и сравнений по модулю $I$ :

$$
\alpha_{j}=f_{j}^{\prime}(a)=\left(f_{1}^{\left[\delta_{1}\right]}\right)^{\prime}(a)=\prod_{k=0}^{\delta_{1}-1} f_{1}^{\prime}\left(f_{1}^{[k]}(a)\right) \equiv \alpha_{1}^{\delta_{1}} \equiv e .
$$

Поэтому $\delta_{j}=2$, и, поскольку $d_{j}>1$, имеем $d_{j}=2$.

Доказанная лемма является аналогом утверждения 9. Теперь сформулируем и докажем аналог утверждения 8. 
Утверждение 11. Пусть $\delta_{1} \neq p, p=2,2 \leq j \leq n-1$ и верно одно из соотношений:

1) $\left(f_{1}^{\left[\delta_{1}\right]}\right)^{\prime}(a) \not \equiv 3 e\left(\bmod I^{2}\right)$

2) $2 t_{1} \mid t_{j}$.

Тогда из равенства $d_{j}=2$ следует, что $d_{s}=2$ для всякого $s \geq j$.

Доказательство. Используется индукция по $s \geq j$. База индукции очевидна.

Осуществим индуктивный переход от $s \geq j$ к $s+1$. По предположению индукции $d_{s}=2$. Тогда $\delta_{s}=2$ и верно соотношение

$$
f_{s}(a) \equiv a+2^{s} y \quad\left(\bmod I^{s+2}\right),
$$

где $y \in R^{*}$. Ввиду (14) получаем:

$$
f_{s+1}(a)=f_{s}^{[2]}(a) \equiv a+2^{s} y\left(e+\alpha_{s}\right) \quad\left(\bmod I^{s+2}\right) .
$$

По лемме 8 и утверждению 7 последовательность

$$
d_{1}, d_{2}, \ldots, d_{s-1}
$$

имеет вид a), b), с) или d), где

a) $1,1, \ldots, 1, \delta_{1}$

b) $1,1, \ldots, 1, \delta_{1}, 1,1, \ldots, 1$;

c) $1,1, \ldots, 1, \delta_{1}, 1,1, \ldots, 1,2$;

d) $1,1, \ldots, 1, \delta_{1}, 1,1, \ldots, 1,2, \varepsilon_{1}, \varepsilon_{2}, \ldots, \varepsilon_{l}$, где $\varepsilon_{i} \in\{1,2\}, i=$ $=1,2, \ldots, l$.

При этом в ситуациях а) и b) верно соотношение $2 t_{1} \nmid t_{s}$, а в ситуациях с) и d) - соотношение $2 t_{1} \mid t_{s}$.

Пусть выполнено условие 2). Тогда $2 t_{1} \mid t_{s}$. Тогда $t_{s}=2^{k} \delta_{1} t_{1}$, где $k \geq 1$. В обозначениях леммы 8 имеем $d_{i}=\delta_{1}$ и

$$
\alpha_{i+1}=\left(f_{1}^{\left[\delta_{1}\right]}\right)^{\prime}(a) \equiv e(\bmod I)
$$

По лемме 5 получаем цепочку равенств и сравнений по модулю $I^{2}$

$$
\alpha_{s}=\left(f_{i+1}^{\left[2^{k}\right]}\right)^{\prime}(a) \equiv\left(\alpha_{i+1}\right)^{2^{k}} \equiv e .
$$


Из (42) получаем

$$
f_{s+1}(a) \equiv a+2^{s+1} y \quad\left(\bmod I^{s+2}\right)
$$

Следовательно, $t_{s+2}>t_{s+1}$. По утверждению 7 из равенства $\delta_{s}=2$ следует, что $\delta_{s+1}=2$. Поэтому $d_{s+1}=2$.

Пусть выполнено условие 1$)$. Случай $2 t_{1} \mid t_{s}$ рассматривается так же, как и выше. Пусть $2 t_{1} \nmid t_{s}$. Тогда

$$
\alpha_{s}=\left(f_{1}^{\left[\delta_{1}\right]}\right)^{\prime}(a) \equiv e(\bmod I)
$$

Отсюда $\alpha_{s} \equiv e+2 r\left(\bmod I^{2}\right)$, где $\bar{r} \neq \bar{e}$. Ввиду (42) имеем следующую цепочку сравнений по модулю $I^{s+2}$ :

$$
f_{s+1}(a) \equiv a+2^{s+1} y(e+r) \not \equiv a .
$$

Поэтому $t_{s+2}>t_{s+1}$. По утверждению 7 из равенства $\delta_{s}=2$ следует, что $\delta_{s+1}=2$. Следовательно, $d_{s+1}=2$. $\triangleright$

По утверждению 10 для рассматриваемого неособого элемента $a \in R$ существует единственный элемент $a^{*} \in R$ такой, что $\bar{a}=\bar{a}^{*}$ и $t_{n}\left(a^{*}\right)=t_{1}\left(a^{*}\right)$. Таким образом, равенство $t_{n}(a)=t_{1}(a)$ выполняется тогда и только тогда, когда $a=a^{*}$. Отсюда из леммы 8 и утверждения 11 вытекает центральный результат этого параграфа.

Теорема 6. Если $\delta_{1} \neq p$ и $p=2$, то последовательность (27) имеет один из следующих видов:
a) $1,1, \ldots, 1$;
b) $1,1, \ldots, 1, \delta_{1}$;
c) $1,1, \ldots, 1, \delta_{1}, 1,1, \ldots, 1$;
d) $1,1, \ldots, 1, \delta_{1}, 1,1, \ldots, 1,2,2, \ldots, 2$;
e) $1,1, \ldots, 1, \delta_{1}, 1,1, \ldots, 1,2,1,1, \ldots, 1$;
f) $1,1, \ldots, 1, \delta_{1}, 1,1, \ldots, 1,2,1,1, \ldots, 1,2,2, \ldots, 2$.

В каждом пункте первая серия единиц может отсутствовать. Ситуацчия а) имеет место тогда и только тогда, когда $a=a^{*}$. Ситуации е) $u$ f) возможны лишь при условии $\left(f_{1}^{\left[\delta_{1}\right]}\right)^{\prime}(a) \equiv 3 e\left(\bmod I^{2}\right)$.

В следующем параграфе будет обсуждаться вопрос: сколько циклов данной длины имеется в графе $G_{F}$ ? 


\section{8. О числе циклов данной длины}

В этом параграфе $R=G R\left(q^{n}, p^{n}\right)$ - произвольное кольцо Галуа (ограничений на $p$ не накладывается). Пусть $a-$ циклическая вершина, и псевдоцикл $C_{F}^{\#}(a)$ неособый. Согласно теореме 1 семейство $C_{F}^{\#}(a)$ распадается на циклы. Исследуем длины этих циклов.

Положим $R_{s}=R / I^{s}, s=1,2, \ldots, n$. Выберем и зафиксируем $s \in\{1,2, \ldots, n-1\}$. Рассмотрим эпиморфизм

$$
\varphi_{s}: R_{s+1} \rightarrow R_{S}
$$

приводящий элементы кольца $R_{S+1}$ по модулю $I^{s}$. Обозначим через $\widetilde{F}$ образ многочлена $F$ при естественном эпиморфизме $R \rightarrow \widetilde{R}=R_{S}$. Если $C-$ некоторый цикл в графе $G_{\widetilde{F}}$, то множество $\varphi_{s}^{-1}(C)$ будем называть прообразом цикла $C$. Цикл длины $t$ будем именовать $t$-цииклом.

Всюду далее будут использоваться следующие краткие обозначения: $f=f_{1}, \alpha=\alpha_{1}, \delta=\delta_{1}$. Пусть вершина $\widetilde{a}$ графа $G_{\widetilde{F}}$ принадлежит циклу $C$. Имеет место

Теорема 7. Пусть $C$ есть t-ичикл. Тогда:

1) если в прообразе ичикла $C$ есть ичикл длины $d t$, где $d \mid q-1 u d>1$, то этот прообраз состоит из одного

2) если в прообразе изкла С есть цикл длины pt, то в этом прообразе $\frac{q}{p}$ ичиклов длины pt;

3) если в прообразе ияила $C$ есть не менее двух прообразе q ииклов длины $t$.

Доказательству теоремы предпошлем лемму.

Лемма 9. Справедливы следуюшие утверждения

1. Если $f(a)=a+p^{s}$ удля некоторых $s \geq 1$ и $y \in R$, то для всякого $x \in R$

$$
t_{s+1}\left(a+p^{s} x\right)=t_{1}(a) \Leftrightarrow \bar{y}=(\bar{e}-\bar{\alpha}) \bar{x} .
$$

2. Eсли $f_{s}(a)=a+p^{s}$ у для некоторых $s \geq 1 u y \in R$, то для всякого $x \in R$

$$
t_{s+1}\left(a+p^{s} x\right)=t_{s}(a) \Leftrightarrow \bar{y}=\left(\bar{e}-\bar{\alpha}_{s}\right) \bar{x} .
$$


Доказательство. Утверждение 1 вытекает из эквивалентных друг другу соотношений

$$
\begin{gathered}
t_{s+1}\left(a+p^{s} x\right)=t_{1}(a) \\
f\left(a+p^{s} x\right) \equiv a+p^{s} x \quad\left(\bmod I^{s+1}\right), \\
a+p^{s} y+p^{s} \alpha x \equiv a+p^{s} x \quad\left(\bmod I^{s+1}\right), \\
p^{s} y \equiv p^{s}(e-\alpha) x \quad\left(\bmod I^{s+1}\right) \\
\bar{y}=(\bar{e}-\bar{\alpha}) \bar{x} .
\end{gathered}
$$

2. Доказательство получается из доказательства утверждения 1 путем замены $t_{1}$ на $t_{s}, f$ на $f_{s}$ и $\alpha$ на $\alpha_{s}$.

Доказательство теоремы 7. 1) По условию $t_{s}(a)=t$, и существует такой элемент $x^{\prime} \in R$, что

$$
t_{s+1}\left(a+p^{s} x^{\prime}\right)=d t
$$

Так как $d \mid q-1$ и $d>1$, по утверждению 7 имеем $\delta \neq p$. Тогда ввиду следствия 4 и теоремы 6

$$
t_{1}(a)=\cdots=t_{s}(a)=t, \quad d=\delta .
$$

Поскольку $\bar{\alpha} \neq \bar{e}$, уравнение относительно $x$

$$
y \equiv(e-\alpha) x \quad(\bmod I)
$$

имеет единственное по модулю $I$ решение $x^{\prime \prime}$. По п. 1 леммы 9, следствию 4 и теореме 6

$$
\forall x \in R: \quad t_{s+1}\left(a+p^{s} x\right)=\delta t \Leftrightarrow x \not \equiv x^{\prime \prime} \quad(\bmod I) .
$$

Это означает, что у вершины $\widetilde{a}$ есть единственный прообраз, лежащий на $t$-цикле, и $q-1$ прообразов, лежащих на $\delta t$-циклах.

На одном $\delta t$-цикле лежит $\delta$ прообразов вершины $\widetilde{a}$ (они расположены на цикле с шагом $\left.t=t_{s}(a)\right)$. Поэтому прообраз цикла $C$ состоит из одного цикла длины $t$ и $\frac{q-1}{\delta}$ циклов длины $\delta t$.

2 ) Пусть в графе $G_{\widetilde{F}}$ вершина $\widetilde{a}$ лежит на $t$-цикле, и в прообразе цикла $C$ есть $p t$-цикл. Достаточно показать, что все прообразы вершины $\widetilde{a}$ лежат на $p t$-циклах. Возможны два случая: $\bar{\alpha}=\bar{e}$ и $\bar{\alpha} \neq \bar{e}$. 
Пусть $\bar{\alpha}=\bar{e}$. При доказательстве утверждения 7 было показано, что в этой ситуации $\bar{\alpha}_{s}=\bar{e}$. По пункту 2 леммы 9 равенство

$$
t_{s+1}\left(a+p^{s} x\right)=t_{s}(a)
$$

имеет место тогда и только тогда, когда $\bar{y}=0$. Однако для некоторого $x^{\prime} \in R$ верно равенство

$$
t_{s+1}\left(a+p^{s} x^{\prime}\right)=p t_{s}(a) .
$$

Это означает, что $\bar{y} \neq 0$, и для любого $x \in R$

$$
t_{s+1}\left(a+p^{s} x\right)>t_{s}(a)
$$

Поскольку $\delta_{s}=p$, всякий прообраз вершины $\widetilde{a}$ лежит на цикле длины $p t$.

Рассмотрим случай $\bar{\alpha} \neq \bar{e}$. Достаточно доказать, что $\bar{\alpha}_{s}=\bar{e}$ (дальнейшее доказательство проводится так же, как в предыдущем случае). Поскольку

$$
t_{s+1}\left(a+p^{s} x^{\prime}\right)=p t_{s}(a)
$$

по следствию 4 и теореме 6 имеем

$$
\delta \mid \Delta=d_{1} d_{2} \ldots d_{s-1} .
$$

Отсюда вытекает цепочка сравнений по модулю $I$

$$
\alpha_{s} \equiv\left(f^{[\Delta]}\right)^{\prime}(a) \equiv\left(f^{\prime}(a)\right)^{\Delta}=\alpha^{\Delta} \equiv e .
$$

3) Пусть существуют такие $x_{1}, x_{2} \in R$, что $\bar{x}_{1} \neq \bar{x}_{2}$ и

$$
t_{s+1}\left(a+p^{s} x_{1}\right)=t_{s}(a)=t_{s+1}\left(a+p^{s} x_{2}\right) .
$$

По пункту 2 леммы 9 имеем

$$
\left(\bar{e}-\bar{\alpha}_{s}\right)\left(\bar{x}_{1}-\bar{x}_{2}\right)=0 .
$$

Отсюда $\bar{e}=\bar{\alpha}_{s}$. Поскольку

$$
t_{s+1}\left(a+p^{s} x_{1}\right)=t_{s}(a),
$$

получаем, что $\bar{y}=0$. Но тогда согласно пункту 2 леммы 9 всякий прообраз элемента $\widetilde{a}$ лежит на цикле длины $t=t_{s}(a)$. Поэтому прообраз цикла $C$ состоит из циклов длины $t$. Количество этих циклов совпадает с количеством прообразов элемента $\widetilde{a}$, то есть с $q . \triangleright$ 


\section{9. Заключение}

В работе исследована цикловая структура полиномиального генератора над кольцом Галуа $R=G R\left(q^{n}, p^{n}\right)$. В частности, доказано, что для произвольного полиномиального преобразования $F$ и любого элемента $a \in R$ имеет место неравенство

$$
t_{n}(a) \leq p^{n-2}(q-1) \cdot t_{1}(a) .
$$

В некоторых ситуациях эта оценка достижима. Путем вычислений на ЭВМ показано, что над кольцами $R_{1}=G R\left(8^{3}, 2^{3}\right)$ и $R_{2}=G R\left(25^{3}, 5^{3}\right)$ существуют пары $(F, a)$, для которых неравенство (43) обращается в равенство. Получены первые нетривиальные результаты о цикловом типе произвольного полиномиального преобразования $F$.

Авторы выражают глубокую признательность профессору А. А. Нечаеву за постановку задачи и внимание к этой работе.

\section{Список литературы}

1. Анашин B.C. О группах и кольцах, обладающих транзитивными полиномами // XVI Всесоюзная алгебраическая конференция. Тезисы. Часть II. - 1981. - C. 4-5.

2. Асанов М. О., Баранский В.А., Расин В. В. Дискретная математика: графы, матроиды, алгоритмы. - Ижевск: НИЦ «Регулярная и хаотическая динамика», 2001. - 288 с.

3. Глухов М. М., Елизаров В. П., Нечаев А. А. Алгебра. - М.: Гелиос, 2003. $749 \mathrm{c}$.

4. Елизаров В. П. Конечные кольца. - М.: Гелиос, 2006. - 304 с.

5. Козлитин O.A. Полиномиальные преобразования GEO-кольца простой характеристики // Дискретная математика. - 2004. - Т. 16. Вып. 3. C. $105-117$.

6. Ларин М.В. Транзитивные полиномиальные преобразования колец вычетов // Дискретная математика. - 2002. - Т. 14. Вып. 2. - С. 20-32.

7. Нечаев А.А. Полиномиальные преобразования конечных коммутативных колец главных идеалов // Математические заметки. - 1980. - Т. 27. Вып. 6. - С. 885-899. 